Bull. Korean Math. Soc. 48 (2011), No. 4, pp. 673-688

DOI 10.4134/BKMS.2011.48.4.673

\title{
ON STABILITY PROBLEMS WITH SHADOWING PROPERTY AND ITS APPLICATION
}

\author{
Hahng-Yun Chu, GilJun Han, and Dong Seung Kang
}

Abstract. Let $n \geq 2$ be an even integer. We investigate that if an odd mapping $f: X \rightarrow Y$ satisfies the following equation

$$
\begin{aligned}
& 2{ }_{n-2} C_{\frac{n}{2}-1} r f\left(\sum_{j=1}^{n} \frac{x_{j}}{r}\right)+\sum_{\substack{i_{k} \in\{0,1\} \\
\sum_{k=1}^{n} i_{k}=\frac{n}{2}}} r f\left(\sum_{i=1}^{n}(-1)^{i_{k}} \frac{x_{i}}{r}\right) \\
= & 2{ }_{n-2} C_{\frac{n}{2}}-1 \sum_{i=1}^{n} f\left(x_{i}\right),
\end{aligned}
$$

then $f: X \rightarrow Y$ is additive, where $r \in \mathbb{R}$. We also prove the stability in normed group by using shadowing property and the Hyers-Ulam stability of the functional equation in Banach spaces and in Banach modules over unital $C^{*}$-algebras. As an application, we show that every almost linear bijection $h: A \rightarrow B$ of unital $C^{*}$-algebras $A$ and $B$ is a $C^{*}$-algebra isomorphism when $h\left(\frac{2^{s}}{r^{s}} u y\right)=h\left(\frac{2^{s}}{r^{s}} u\right) h(y)$ for all unitaries $u \in A$, all $y \in A$, and $s=0,1,2, \ldots$.

\section{Introduction}

In 1940, the problem of stability of functional equations was originated by Ulam [15] as follows: Under what condition does there exist an additive mapping near an approximately additive mapping?

The first partial solution to Ulam's question was provided by D. H. Hyers [7]. Let $X$ and $Y$ be Banach spaces with norms $\|\cdot\|_{X}$ and $\|\cdot\|_{Y}$, respectively. Hyers showed that if a function $f: X \rightarrow Y$ satisfies the following inequality

$$
\|f(x+y)-f(x)-f(y)\|_{Y} \leq \epsilon
$$

Received June 2, 2009; Revised January 11, 2010.

2010 Mathematics Subject Classification. 39B52.

Key words and phrases. shadowing property, Hyers-Ulam-Rassias stability, additive mapping, $\mathrm{C}^{*}$-algebra isomorphism.

This research was supported by Basic Science Research Program through the National Research Foundation of Korea(NRF) funded by the Ministry of Education, Science and Technology(2010-0013784). 
for all $\epsilon \geq 0$ and for all $x, y \in X$, then the limit

$$
a(x)=\lim _{n \rightarrow \infty} 2^{-n} f\left(2^{n} x\right)
$$

exists for each $x \in X$ and $a: X \rightarrow Y$ is the unique additive function such that

$$
\|f(x)-a(x)\|_{Y} \leq \epsilon
$$

for any $x \in X$. Moreover, if $f(t x)$ is continuous in $t$ for each fixed $x \in X$, then $a$ is linear.

Hyers' theorem was generalized in various directions. In particular, thirty seven years after Hyers' Theorem, Th. M. Rassias provided a generalization of Hyers' result by allowing for the first time in the subject of functional equations and inequalities the Cauchy difference to be unbounded; see [11]. Găvruta [6] provided a generalization of the Theorem of Th. M. Rassias.

Several functional equations have been investigated in [3], [4], [5]. Recently, Bae and Park investigated that the generalized Hyers-Ulam-Rassias stability in Banach modules over a $C^{*}$-algebra and unitary Banach algebra; see [1]. In [2], Baak et al. introduced generalized additive functional equation and studied the stability in Banach modules and isomorphisms between $C^{*}$-algebras. Recently, Lee, Koh and $\mathrm{Ku}[10]$ investigated the stability via shadowing property with a quadratic functional equation.

Let $n$ be an even integer, and $r \in \mathbb{R}$. In this paper, we investigate that a mapping $f: X \rightarrow Y$ satisfies the following equation

$$
\begin{aligned}
& 2{ }_{n-2} C_{\frac{n}{2}-1} r f\left(\sum_{j=1}^{n} \frac{x_{j}}{r}\right)+\sum_{\substack{i_{k} \in\{0,1\} \\
\sum_{k=1}^{n} i_{k}=\frac{n}{2}}} r f\left(\sum_{i=1}^{n}(-1)^{i_{k}} \frac{x_{i}}{r}\right) \\
= & 2{ }_{n-2} C_{\frac{n}{2}-1} \sum_{i=1}^{n} f\left(x_{i}\right) .
\end{aligned}
$$

First of all, we show that if above mapping $f$ is odd, then it is additive. Next, we prove the stability in normed group by using shadowing property. By using the some results as in [2], we study the Hyers-Ulam stability of the functional equation in Banach spaces and also in Banach modules over a unital $C^{*}$-algebras. Also, its application, we show that every almost linear bijection $h: A \rightarrow B$ of unital $C^{*}$-algebras $A$ and $B$ is a $C^{*}$-algebra isomorphism when $h\left(\frac{2^{s}}{r^{s}} u y\right)=h\left(\frac{2^{s}}{r^{s}} u\right) h(y)$ for all unitaries $u \in A$, all $y \in A$, and $s=0,1,2, \ldots$ 


\section{Generalized additive mapping in several variables}

Lemma 2.1. Let $n \geq 2$ be an even integer number, and let $X, Y$ be vector spaces. A given odd mapping $f: X \rightarrow Y$ defined by

$$
\begin{aligned}
& 2{ }_{n-2} C_{\frac{n}{2}-1} r f\left(\sum_{j=1}^{n} \frac{x_{j}}{r}\right)+\sum_{\substack{i_{k} \in\{0,1\} \\
\sum_{k=1}^{n} i_{k}=\frac{n}{2}}} r f\left(\sum_{i=1}^{n}(-1)^{i_{k}} \frac{x_{i}}{r}\right) \\
= & 2{ }_{n-2} C_{\frac{n}{2}-1} \sum_{i=1}^{n} f\left(x_{i}\right),
\end{aligned}
$$

is additive, for all $x_{1}, \ldots, x_{n} \in X$.

Proof. Since $f$ is odd, we have $f(0)=0$.

Now, by letting $x_{1}=x, x_{2}=y$, and $x_{3}=\cdots=x_{n}=0$, we get

$$
\begin{aligned}
& 2{ }_{n-2} C_{\frac{n}{2}-1} r f\left(\frac{x+y}{r}\right)+{ }_{n-2} C_{\frac{n}{2}} r f\left(\frac{x+y}{r}\right)+{ }_{n-2} C_{\frac{n}{2}-1} r f\left(\frac{-x+y}{r}\right) \\
& +{ }_{n-2} C_{\frac{n}{2}-1} r f\left(\frac{x-y}{r}\right)+{ }_{n-2} C_{\frac{n}{2}-2} r f\left(\frac{-x-y}{r}\right) \\
= & 2{ }_{n-2} C_{\frac{n}{2}-1}(f(x)+f(y))
\end{aligned}
$$

for all $x, y \in X$. Since $f$ is odd and ${ }_{n-2} C_{\frac{n}{2}-2}={ }_{n-2} C_{\frac{n}{2}}$, we have

$$
2{ }_{n-2} C_{\frac{n}{2}-1} r f\left(\frac{x+y}{r}\right)=2{ }_{n-2} C_{\frac{n}{2}-1}(f(x)+f(y))
$$

for all $x, y \in X$. Then we get

$$
r f\left(\frac{x+y}{r}\right)=f(x)+f(y)
$$

for all $x, y \in X$. Hence we have $r f\left(\frac{x}{r}\right)=f(x)$, when $y=0$. Thus $f(x+y)=$ $r f\left(\frac{x+y}{r}\right)=f(x)+f(y)$, that is, $f$ is additive.

\section{Stability using shadowing property}

In this section, we will investigate the stability of the given functional equation based on the ideas from dynamical systems. Before we proceed, we would like to introduce some basic definitions concerning shadowing and key concepts to establish the stability; see [14].

Let us fix some notations which will be used throughout this section. First of all, we will fix $r=1$, that is, we will investigate the generalized mappings of 1-type. Also, we denote $\mathbb{N}$ the set of all nonnegative integers, $X$ a complete normed space and $B(x, s)$ the closed ball centered at $x$ with radius $s$ and let $\phi: X \rightarrow X$ be given. 
Definition 3.1. Let $\delta \geq 0$. We say that a sequence $\left(x_{k}\right)_{k \in \mathbb{N}}$ is a $\delta$-pseudoorbit $($ for $\phi)$ if

$$
d\left(x_{k+1}, \phi\left(x_{k}\right)\right) \leq \delta \quad \text { for } k \in \mathbb{N} .
$$

A 0-pseudoorbit is called an orbit.

Definition 3.2. Let $s, R>0$ be given. We say that $\phi: X \rightarrow X$ is locally $(s, R)$-invertible at $x_{0} \in X$ if

$$
\forall y \in B\left(\phi\left(x_{0}\right), R\right), \quad \exists ! x \in B\left(x_{0}, s\right): \quad \phi(x)=y .
$$

If $\phi$ is locally $(s, R)$-invertible at each $x \in X$, then we say that $\phi$ is locally $(s, R)$-invertible.

For a locally $(s, R)$-invertible function $\phi$, we define a function

$$
\phi_{x_{0}}^{-1}: B\left(\phi\left(x_{0}\right), R\right) \rightarrow B\left(x_{0}, s\right)
$$

in such a way that $\phi_{x_{0}}^{-1}(y)$ denote the unique $x$ from the above definition which satisfies $\phi(x)=y$. Moreover, we put

$$
\operatorname{lip}_{R} \phi^{-1}:=\sup _{x_{0} \in X} \operatorname{lip}\left(\phi_{x_{0}}^{-1}\right)
$$

where $\operatorname{lip}\left(\phi_{x_{0}}^{-1}\right)$ is the lipschitz constant of $\phi_{x_{0}}^{-1}$.

Theorem 3.3 ([13]). Let $l \in(0,1), R \in(0, \infty)$ be fixed and let $\phi: X \rightarrow X$ be locally $(l R, R)$-invertible. We assume additionally that $\operatorname{lip}_{R}\left(\phi^{-1}\right) \leq l$. Let $\delta \leq(1-l) R$ and let $\left(x_{k}\right)_{k \in \mathbb{N}}$ be an arbitrary $\delta$-pseudoorbit. Then there exists a unique $y \in X$ such that

$$
d\left(x_{k}, \phi^{k}(y)\right) \leq l R \quad \text { for } k \in \mathbb{N} .
$$

Moreover,

$$
d\left(x_{k}, \phi^{k}(y)\right) \leq \frac{l \delta}{1-l} \quad \text { for } k \in \mathbb{N}
$$

Let $(X, *)$ be a semigroup. We denote $k x$ to be $\underbrace{x * \cdots * x}_{k}$, where $x \in X$ and $k \in \mathbb{N}$. Then the mapping $\|\cdot\|: X \rightarrow \mathbb{R}$ is called a (semigroup) norm if it satisfies the following properties:

(1) for all $x \in X,\|x\| \geq 0$.

(2) for all $x \in X, k \in \mathbb{N},\|k x\|=k\|x\|$.

(3) for all $x, y \in X,\|x\|+\|y\| \geq\|x * y\|$ and also the equality holds when $x=y$, where $*$ is the binary operation on $X$.

Note $(X, *,\|\cdot\|)$ is called a normed group if $X$ is a group with an identity $e$, and it additionally satisfies that $\|x\|=0$ if and only if $x=e$.

We say that $(X, *,\|\cdot\|)$ is a normed (semi)group if $X$ is a (semi)group with a norm $\|\cdot\|$. Now, given an Abelian group $X$ and $n \in \mathbb{Z}$, we define the mapping $\left[n_{X}\right]: X \rightarrow X$ by the formula

$$
\left[n_{X}\right](x):=n x \quad \text { for } x \in X .
$$


Also, we are going to need the following result. In recent years, Lee et al. showed the next lemma by using Theorem 3.3.

Lemma $3.4([10])$. Let $l \in(0,1), R \in(0, \infty), \delta \in(0,(1-l) R), \varepsilon>0, m \in$ $\mathbb{N}, n \in \mathbb{Z}$. Let $G$ be a commutative semigroup, $X$ a complete Abelian metric group. We assume that the mapping $\left[n_{X}\right]$ is locally $(l R, R)$-invertible and that $\operatorname{lip}_{R}\left(\left[n_{X}\right]^{-1}\right) \leq l$. Let $f: G \rightarrow X$ satisfy the following two inequalities

$$
\begin{aligned}
\left\|\sum_{i=1}^{N} a_{i} f\left(b_{i_{1}} x_{1}+\cdots+b_{i_{n}} x_{n}\right)\right\| & \leq \varepsilon \quad \text { for } x_{1}, \ldots, x_{n} \in G, \\
\|f(m x)-n f(x)\| & \leq \delta \quad \text { for } x \in G,
\end{aligned}
$$

where all $a_{i}$ are endomorphisms in $X$ and $b_{i_{j}}$ are endomorphisms in $G$. We assume additionally that there exists $K \in\{1, \ldots, N\}$ such that

$$
\sum_{i=1}^{K} \operatorname{lip}\left(a_{i}\right) \delta \leq(1-l) R, \quad \varepsilon+\sum_{i=K+1}^{N} \operatorname{lip}\left(a_{i}\right) \frac{l \delta}{1-l} \leq l R .
$$

Then there exists a unique function $F: G \rightarrow X$ such that

$$
F(m x)=n F(x) \quad \text { for } x \in G,
$$

and

$$
\|f(x)-F(x)\| \leq \frac{l \delta}{1-l} \quad \text { for } x \in G .
$$

Moreover, F satisfies

$$
\sum_{i=1}^{N} a_{i} F\left(b_{i_{1}} x_{1}+\cdots+b_{i_{n}} x_{n}\right)=0 \quad \text { for } x_{1}, \ldots, x_{n} \in G .
$$

Now, we are ready to prove our functional equations as follows:

$$
\begin{aligned}
D f\left(x_{1}, \ldots, x_{n}\right):= & 2{ }_{n-2} C_{\frac{n}{2}-1} f\left(\sum_{j=1}^{n} x_{j}\right) \\
& +\sum_{\substack{i_{k} \in\{0,1\} \\
\sum_{k=1}^{n} i_{k}=\frac{n}{2}}} f\left(\sum_{k=1}^{n}(-1)^{i_{k}} x_{k}\right) \\
& -2_{n-2} C_{\frac{n}{2}-1} \sum_{i=1}^{n} f\left(x_{i}\right)
\end{aligned}
$$

for all $x_{1}, \ldots, x_{n} \in G$.

Theorem 3.5. Let $R>0$, let $n \geq 3$ be an even integer, $G$ a commutative semigroup, and let $X$ be a complete normed Abelian group. Suppose that $\left[n_{X}\right]$ is locally $\left(\frac{R}{4}, R\right)$-invertible and $\operatorname{lip}_{R}\left(\left[n_{X}\right]^{-1}\right) \leq \frac{1}{4}$. Let $\varepsilon \leq \frac{3(n-2)}{4 n(5 n-4)} R$ and let $f: G \rightarrow X$ be a function such that

$$
\left\|D f\left(x_{1}, \ldots, x_{n}\right)\right\| \leq \varepsilon
$$


for all $x_{1}, \ldots, x_{n}, x \in G$. Then there exists a unique function $F: G \rightarrow X$ such that

$$
\begin{aligned}
& F(n x)=n F(x), \\
& D F\left(x_{1}, \ldots, x_{n}\right)=0 \\
& \|F(x)-f(x)\| \leq \frac{n}{6(n-2)_{n-2} C_{\frac{n}{2}-1}} \varepsilon
\end{aligned}
$$

for all $x_{1}, \ldots, x_{n}, x \in G$.

Proof. By letting $x_{1}=\cdots=x_{n}=0$ in the equation (3.3), we have

$$
\left\|\frac{n-2}{2}{ }_{n} C_{\frac{n}{2}} f(0)\right\| \leq \varepsilon,
$$

that is, $\|f(0)\| \leq \frac{2 \varepsilon}{(n-2)_{n} C_{\frac{n}{2}}}$. Now, by putting $x_{k}=x(k=1, \ldots, n)$ in $(3.3)$, the inequality $\|f(0)\| \leq \frac{2 \varepsilon}{(n-2)_{n} C_{\frac{n}{2}}}$ implies

$$
\left\|2_{n-2} C_{\frac{n}{2}-1} f(n x)-2 n_{n-2} C_{\frac{n}{2}-1} f(x)\right\| \leq \frac{n}{n-2} \varepsilon,
$$

that is, we have $\|f(n x)-n f(x)\| \leq \frac{n}{2(n-2)_{n-2} C_{\frac{n}{2}-1}} \varepsilon$ for all $x \in G$. To apply Lemma 3.4 for the function $f$, we may let

$$
\begin{array}{r}
l=\frac{1}{4}, \delta=\frac{n}{2(n-2)_{n-2} C_{\frac{n}{2}-1}} \varepsilon, K={ }_{n} C_{\frac{n}{2}}, \\
a_{1}=\cdots=a_{K}=\mathrm{id}_{X}, \\
a_{K+1}=\cdots=a_{K+n}=-2_{n-2} C_{\frac{n}{2}-1} \mathrm{id}_{X}, \\
a_{N}=2_{n-2} C_{\frac{n}{2}-1} \mathrm{id}_{X}, \text { where } N=K+n+1 .
\end{array}
$$

Then we have

$$
\begin{aligned}
\delta & =\frac{n}{2(n-2)_{n-2} C_{\frac{n}{2}-1}} \varepsilon \\
& \leq \frac{n}{2(n-2)_{n-2} C_{\frac{n}{2}-1}} \cdot \frac{3(n-2)}{4 n(5 n-4)} R \\
& \leq \frac{3}{4} R=(1-l) R \\
\sum_{i=1}^{K} \operatorname{lip}\left(a_{i}\right) \delta & =K \cdot \frac{n}{2(n-2)_{n-2} C_{\frac{n}{2}-1}} \varepsilon \\
& \leq \frac{2(n-1)}{n(5 n-4)} \cdot \frac{3}{4} R \\
& \leq \frac{3}{4} R=(1-l) R
\end{aligned}
$$


and

$$
\begin{aligned}
\varepsilon+\sum_{i=K+1}^{N} \operatorname{lip}\left(a_{i}\right) \frac{l \delta}{1-l} & =\varepsilon+\frac{1}{3} \cdot \frac{n(n+1)}{n-2} \varepsilon \\
& \leq \frac{n^{2}+4 n-6}{4 n(5 n-4)} R \\
& \leq \frac{1}{4} R=l R .
\end{aligned}
$$

Hence all conditions of Lemma 3.4 are satisfied, and thus we conclude that there exists a unique function $F: G \rightarrow X$ such that

$$
\begin{array}{r}
F(n x)=n F(x), \\
D F\left(x, \ldots, x_{n}\right)=0
\end{array}
$$

and also we have

$$
\|f(x)-F(x)\| \leq \frac{l \delta}{1-l}=\frac{n}{6(n-2)_{n-2} C_{\frac{n}{2}-1}} \varepsilon \text { for all } x_{1}, \ldots, x_{n}, x \in G .
$$

Theorem 3.6. Let $R>0$, let $n \geq 3$ be an even integer, $G$ a commutative semigroup, $X$ a complete normed Abelian group, and let $f: G \rightarrow X$ be a function. Suppose that $\left[\left({ }_{n-1} C_{\frac{n}{2}}\right)_{X}\right]$ is locally $\left(\frac{R}{{ }_{n-1} C_{\frac{n}{2}}}, R\right)$-invertible, $\left[\left(\frac{1}{2}(n-2)_{n} C_{\frac{n}{2}}\right)_{X}\right]$ is locally $\left(\frac{2 R}{(n-2)_{n} C_{\frac{n}{2}}}, R\right)$-invertible, and $\left[\left(2_{n-2} C_{\frac{n}{2}-1}\right)_{X}\right]$ is locally $\left(\frac{R}{2_{n-2} C_{\frac{n}{2}-1}}, R\right)$ invertible. Then $f$ satisfies the following equation

$$
\operatorname{Df}\left(x_{1}, \ldots, x_{n}\right)=0
$$

for all $x_{1}, \ldots, x_{n} \in G$ if and only if $f$ is a Cauchy additive odd function.

Proof. We are going to prove that $f$ as in the equation (3.5) is a Cauchy additive mapping. By letting $x_{k}=0(k=1, \ldots, n)$ in the equation $(3.5)$, we have

$$
\frac{n-2}{2}{ }_{n} C_{\frac{n}{2}} f(0)=0 \text {. }
$$

By the uniqueness of the local division by $\frac{n-2}{2}{ }_{n} C_{\frac{n}{2}}$, we get $f(0)=0$.

Also, setting $x_{1}=x, x_{k}=0(k=2, \ldots, n)$ and by the uniqueness of the local division by ${ }_{n-1} C_{\frac{n}{2}}$, the equation $f(0)=0$ implies that

$$
2{ }_{n-2} C_{\frac{n}{2}-1} f(x)+{ }_{n-1} C_{\frac{n}{2}} f(x)+{ }_{n-1} C_{\frac{n}{2}-1} f(-x)=2{ }_{n-2} C_{\frac{n}{2}-1} f(x),
$$

that is, we have $-f(x)=f(-x)$ for all $x \in G$.

Now, by letting $x_{1}=x, x_{2}=y$, and $x_{3}=\cdots=x_{n}=0$, we get

$$
\begin{aligned}
& 2{ }_{n-2} C_{\frac{n}{2}-1} f(x+y)+{ }_{n-2} C_{\frac{n}{2}} f(x+y)+{ }_{n-2} C_{\frac{n}{2}-1} f(-x+y) \\
& +{ }_{n-2} C_{\frac{n}{2}-1} f(x-y)+{ }_{n-2} C_{\frac{n}{2}-2} f(-x-y) \\
= & 2{ }_{n-2} C_{\frac{n}{2}-1}(f(x)+f(y))
\end{aligned}
$$

for all $x, y \in X$. 

have

By the uniqueness of the local division by $2{ }_{n-2} C_{\frac{n}{2}-1}$ and odd property, we

$$
f(x+y)=f(x)+f(y)
$$

for all $x, y \in G$, that is, $f$ is a Cauchy additive mapping, as desired. Conversely, suppose that $f$ is a Cauchy additive odd function. Then

$$
\begin{aligned}
& 2_{n-2} C_{\frac{n}{2}-1} f\left(\sum_{j=1}^{n} x_{j}\right)+\sum_{\substack{i_{k} \in\{0,1\} \\
\sum_{k=1}^{n} i_{k}=\frac{n}{2}}} f\left(\sum_{i=1}^{n}(-1)^{i_{k}} x_{i}\right) \\
= & 2_{n-2} C_{\frac{n}{2}-1} f\left(\sum_{j=1}^{n} x_{j}\right)+0 \\
= & 2_{n-2} C_{\frac{n}{2}-1}\left(f\left(x_{1}\right)+\cdots+f\left(x_{n}\right)\right) \\
= & 2_{n-2} C_{\frac{n}{2}-1} \sum_{i=1}^{n} f\left(x_{i}\right)
\end{aligned}
$$

for all $x_{1}, \ldots, x_{n} \in G$. Thus $f$ satisfies the equation (3.5).

The direct application of Theorems 3.5 and 3.6 yields the following corollary.

Corollary 3.7. Let $R>0$, let $n \geq 3$ be an even integer, $G$ an Abelian group, and $X$ a complete normed Abelian group. Let $\varepsilon \leq \frac{3(n-2)}{4 n(5 n-4)} R$ be arbitrary and $f: G \rightarrow X$ a function satisfying the equation (3.3). Suppose that $\left[n_{X}\right]$ is locally $\left(\frac{R}{4}, R\right)$-invertible with $\operatorname{lip}_{R}\left(\left[n_{X}\right]^{-1}\right) \leq \frac{1}{4}$, and $\left[\left({ }_{n-1} C_{\frac{n}{2}}\right)_{X}\right]$ is locally $\left(\frac{R}{n-1 C_{\frac{n}{2}}}, R\right)$-invertible, $\left[\left(\frac{1}{2}(n-2) n C_{\frac{n}{2}}\right)_{X}\right]$ is locally $\left(\frac{2 R}{(n-2) n C_{\frac{n}{2}}}, R\right)$-invertible, and $\left[\left(2_{n-2} C_{\frac{n}{2}-1}\right)_{X}\right]$ is locally $\left(\frac{R}{2_{n-2} C_{\frac{n}{2}}-1}, R\right)$-invertible. Then there exists a Cauchy additive odd function $F: G \rightarrow X$ such that

$$
\|F(x)-f(x)\| \leq \frac{n}{6(n-2)_{n-2} C_{\frac{n}{2}-1}}
$$

for all $x \in G$.

\section{On Hyers-Ulam-Rassias stabilities}

From now on, let $X$ be a normed vector space with norm $\|\cdot\|_{X}$ and $Y$ be a Banach space with norm $\|\cdot\|_{Y}$. Let $n \geq 2$ be even. 
For the given mapping $f: X \rightarrow Y$, we define

$$
\begin{aligned}
D f\left(x_{1}, \ldots, x_{n}\right):= & 2{ }_{n-2} C_{\frac{n}{2}-1} r f\left(\sum_{j=1}^{n} \frac{x_{j}}{r}\right) \\
& +\sum_{\substack{i_{k} \in\{0,1\} \\
\sum_{k=1}^{n} i_{k}=\frac{n}{2}}} \operatorname{rf}\left(\sum_{k=1}^{n}(-1)^{i_{k}} \frac{x_{k}}{r}\right) \\
& -2_{n-2} C_{\frac{n}{2}-1} \sum_{i=1}^{n} f\left(x_{i}\right)
\end{aligned}
$$

for all $x_{1}, \ldots, x_{n} \in X$.

Theorem 4.1. Let $n \geq 2$ be even and let $f: X \rightarrow Y$ be an odd mapping for which there exists a function $\phi: X^{n} \rightarrow[0, \infty)$ such that

$$
\begin{gathered}
\widetilde{\phi}\left(x_{1}, \ldots, x_{n}\right):=\sum_{j=0}^{\infty}\left(\frac{r}{2}\right)^{j} \phi\left(\left(\frac{2}{r}\right)^{j} x_{1}, \ldots,\left(\frac{2}{r}\right)^{j} x_{n}\right)<\infty, \\
\left\|D f\left(x_{1}, \ldots, x_{n}\right)\right\|_{Y} \leq \phi\left(x_{1}, \ldots, x_{n}\right)
\end{gathered}
$$

for all $x_{1}, \ldots, x_{n} \in X$. Then there exists a unique generalized additive mapping $L: X \rightarrow Y$ such that

$$
\|f(x)-L(x)\|_{Y} \leq \frac{1}{4 \cdot n-2} C_{\frac{n}{2}-1} \widetilde{\phi}(x, x, 0, \ldots, 0)
$$

for all $x \in X$.

Proof. By letting $x_{1}=x_{2}=x$ and $x_{j}=0(j=3, \ldots, n)$ in $(4.3)$, since $f$ is an odd mapping, we have

$$
\left\|2_{n-2} C_{\frac{n}{2}-1} r f\left(\frac{2}{r} x\right)-4{ }_{n-2} C_{\frac{n}{2}-1} f(x)\right\|_{Y} \leq \phi(x, x, 0, \ldots, 0)
$$

for all $x \in X$. Then we obtain that

$$
\left\|f(x)-\frac{r}{2} f\left(\frac{2}{r} x\right)\right\|_{Y} \leq \frac{1}{4 \cdot{ }_{n-2} C_{\frac{n}{2}-1}} \phi(x, x, 0, \ldots, 0)
$$

for all $x \in X$.

From the equation (4.5), we have

$$
\begin{array}{r}
\left\|\left(\frac{r}{2}\right)^{d} f\left(\left(\frac{2}{r}\right)^{d} x\right)-\left(\frac{r}{2}\right)^{d+1} f\left(\left(\frac{2}{r}\right)^{d+1} x\right)\right\|_{Y} \\
\leq\left(\frac{r}{2}\right)^{d} \cdot \frac{1}{4} \cdot \frac{1}{{ }_{n-2} C_{\frac{n}{2}-1}} \phi\left(\left(\frac{2}{r}\right)^{d} x,\left(\frac{2}{r}\right)^{d} x, 0, \ldots, 0\right)
\end{array}
$$


for all $x \in X$ and all positive integer $d$. Hence we get

$$
\begin{aligned}
& \left\|\left(\frac{r}{2}\right)^{s} f\left(\left(\frac{2}{r}\right)^{s} x\right)-\left(\frac{r}{2}\right)^{d} f\left(\left(\frac{2}{r}\right)^{d} x\right)\right\|_{Y} \\
\leq & \frac{1}{4} \cdot \frac{1}{{ }_{n-2} C_{\frac{n}{2}-1}} \sum_{j=s}^{d-1}\left(\frac{r}{2}\right)^{j} \phi\left(\left(\frac{2}{r}\right)^{j} x,\left(\frac{2}{r}\right)^{j} x, 0, \ldots, 0\right)
\end{aligned}
$$

for all $x \in X$ and all positive integers $s$ and $d$ with $s<d$. Hence we may conclude that the sequence $\left\{\left(\frac{r}{2}\right)^{s} f\left(\left(\frac{2}{r}\right)^{s} x\right)\right\}$ is a Cauchy sequence. Hence the sequence converges in $Y$ for all $x \in X$. Thus we may define a mapping $L: X \rightarrow$ $Y$ via

$$
L(x)=\lim _{s \rightarrow \infty}\left(\frac{r}{2}\right)^{s} f\left(\left(\frac{2}{r}\right)^{s} x\right)
$$

for all $x \in X$. Since $f$ is odd, so is $L$. Then by the definition of $D L\left(x_{1}, \ldots, x_{n}\right)$ and (4.3),

$$
\left\|D L\left(x_{1}, \ldots, x_{n}\right)\right\|_{Y} \leq \lim _{s \rightarrow \infty}\left(\frac{r}{2}\right)^{s} \phi\left(\left(\frac{2}{r}\right)^{s} x_{1}, \ldots,\left(\frac{2}{r}\right)^{s} x_{n}\right)=0
$$

for all $x_{1}, \ldots, x_{n} \in X$. That is, $D L\left(x_{1}, \ldots, x_{n}\right)=0$. By Lemma 2.1, the mapping $L: X \rightarrow Y$ is a generalized additive mapping of $r$-type. Also, letting $s=0$ and passing the limit $d \rightarrow \infty$ in (4.6), we get the equation (4.4).

Now, let $L^{\prime}: X \rightarrow Y$ be another generalized additive mapping of $r$-type satisfying (4.4). Then we have

$$
\begin{aligned}
& \left\|L(x)-L^{\prime}(x)\right\|_{Y}=\left(\frac{r}{2}\right)^{s}\left\|L\left(\left(\frac{2}{r}\right)^{s} x\right)-L^{\prime}\left(\left(\frac{2}{r}\right)^{s} x\right)\right\|_{Y} \\
\leq & \left(\frac{r}{2}\right)^{s}\left(\left\|L\left(\left(\frac{2}{r}\right)^{s} x\right)-f\left(\left(\frac{2}{r}\right)^{s} x\right)\right\|_{Y}+\left\|L^{\prime}\left(\left(\frac{2}{r}\right)^{s} x\right)-f\left(\left(\frac{2}{r}\right)^{s} x\right)\right\|_{Y}\right) \\
= & \frac{1}{2} \frac{1}{{ }_{n-2} C_{\frac{n}{2}-1}} \sum_{j=s}^{\infty}\left(\frac{r}{2}\right)^{j} \phi\left(\left(\frac{2}{r}\right)^{j} x,\left(\frac{2}{r}\right)^{j} x, 0, \ldots, 0\right) \rightarrow 0
\end{aligned}
$$

for all $x \in X$ as $s \rightarrow \infty$. Thus we may conclude that such a generalized additive mapping $L$ is unique.

Theorem 4.2. Let $n \geq 2$ be even and let $f: X \rightarrow Y$ be an odd mapping for which there exists a function $\phi: X^{n} \rightarrow[0, \infty)$ such that

$$
\begin{gathered}
\widetilde{\phi}\left(x_{1}, \ldots, x_{n}\right):=\sum_{j=1}^{\infty}\left(\frac{2}{r}\right)^{j} \phi\left(\left(\frac{r}{2}\right)^{j} x_{1}, \ldots,\left(\frac{r}{2}\right)^{j} x_{n}\right)<\infty, \\
\left\|D f\left(x_{1}, \ldots, x_{n}\right)\right\|_{Y} \leq \phi\left(x_{1}, \ldots, x_{n}\right)
\end{gathered}
$$


for all $x_{1}, \ldots, x_{n} \in X$. Then there exists a unique generalized additive mapping $L: X \rightarrow Y$ such that

$$
\|f(x)-L(x)\|_{Y} \leq \frac{1}{4_{n-2} C_{\frac{n}{2}-1}} \widetilde{\phi}(x, x, 0, \ldots, 0)
$$

for all $x \in X$.

Proof. Replacing $x$ by $\frac{r}{2} x$ in the equation (4.5), we have

$$
\left\|f(x)-\frac{2}{r} f\left(\frac{r}{2} x\right)\right\|_{Y} \leq \frac{1}{4_{n-2} C_{\frac{n}{2}-1}} \frac{2}{r} \phi\left(\frac{r}{2} x, \frac{r}{2} x, 0, \ldots, 0\right) .
$$

The remains follow from the proof of Theorem 4.1.

\section{Isomorphisms between unital $C^{*}$-algebras}

In this section, we are going to investigate $C^{*}$-algebra isomorphisms between a unital $C^{*}$-algebra. Hence, first of all, we will prove that the Hyers-Ulam stability of the given functional equation below in a Banach module over a unital $C^{*}$-algebra.

Assume that $A$ is a unital $C^{*}$-algebra with norm $|\cdot|$ and unital group $U(A)$, and that $X$ and $Y$ are left Banach modules over a unital $C^{*}$-algebra $A$ with norms $\|\cdot\|_{X}$ and $\|\cdot\|_{Y}$, respectively.

Given a mapping $f: X \rightarrow Y$, we set

$$
\begin{aligned}
D_{u} f\left(x_{1}, \ldots, x_{n}\right):= & 2{ }_{n-2} C_{\frac{n}{2}-1} r f\left(\sum_{j=1}^{n} \frac{u x_{j}}{r}\right) \\
& +\sum_{\substack{i_{k} \in\{0,1\} \\
\sum_{k=1}^{n} i_{k}=\frac{n}{2}}} r f\left(\sum_{k=1}^{n}(-1)^{i_{k}} \frac{u x_{k}}{r}\right) \\
& -2_{n-2} C_{\frac{n}{2}-1} \sum_{i=1}^{n} u f\left(x_{i}\right)
\end{aligned}
$$

for all $u \in U(A)$ and all $x_{1}, \ldots, x_{n} \in X$.

Theorem 5.1. Let $f: X \rightarrow Y$ be an odd mapping for which there exists a function $\phi: X^{n} \rightarrow[0, \infty)$ such that

$$
\begin{gathered}
\widetilde{\phi}\left(x_{1}, \ldots, x_{n}\right):=\sum_{j=0}^{\infty}\left(\frac{r}{2}\right)^{j} \phi\left(\left(\frac{2}{r}\right)^{j} x_{1}, \ldots,\left(\frac{2}{r}\right)^{j} x_{n}\right)<\infty, \\
\left\|D_{u} f\left(x_{1}, \ldots, x_{n}\right)\right\|_{Y} \leq \phi\left(x_{1}, \ldots, x_{n}\right)
\end{gathered}
$$

for all $u \in U(A)$ and all $x_{1}, \ldots, x_{n} \in X$. Then there exists a unique generalized A-linear mapping $L: X \rightarrow Y$ such that

$$
\|f(x)-L(x)\|_{Y} \leq \frac{1}{4 \cdot_{n-2} C_{\frac{n}{2}-1}} \widetilde{\phi}(x, x, 0, \ldots, 0)
$$


for all $x \in X$.

Proof. Let $u=1 \in U(A)$. By Theorem 4.1, we know that there exists a unique generalized additive mapping satisfying the equation (5.3) defined by

$$
L(x):=\lim _{s \rightarrow \infty} \frac{r^{s}}{2^{s}} f\left(\frac{2^{s}}{r^{s}} x\right)
$$

for all $x \in X$. Also, $L(x)$ is odd.

Now, by assumption for each $u \in U(A)$, we get

$$
\begin{aligned}
\left\|D_{u} L(x, 0, \ldots, 0)\right\|_{Y} & =\lim _{s \rightarrow \infty} \frac{r^{s}}{2^{s}}\left\|D_{u} f\left(\frac{2^{s}}{r^{s}} x, 0, \ldots, 0\right)\right\|_{Y} \\
& \leq \lim _{s \rightarrow \infty} \frac{r^{s}}{2^{s}} \phi\left(\frac{2^{s}}{r^{s}} x, 0, \ldots, 0\right)=0
\end{aligned}
$$

for all $x \in X$. Also, we have

$$
\begin{aligned}
& 2_{n-2} C_{\frac{n}{2}-1} r L\left(\frac{u}{r} x\right)+{ }_{n-1} C_{\frac{n}{2}-1} r L\left(-\frac{u}{r} x\right)+{ }_{n-1} C_{\frac{n}{2}} r L\left(\frac{u}{r} x\right) \\
= & 2{ }_{n-2} C_{\frac{n}{2}-1} u L(x)
\end{aligned}
$$

for all $u \in U(A)$ and all $x \in X$. Since ${ }_{n-1} C_{\frac{n}{2}-1}={ }_{n-1} C_{\frac{n}{2}}$, and $L$ is odd and additive function, we have

$$
r L\left(\frac{u}{r} x\right)=u L(x)
$$

for all $u \in U(A)$ and all $x \in X$.

Now, let $a \in A$ and $M$ an integer greater than $4|a|$. Then

$$
\left|\frac{a}{M}\right|<\frac{1}{4}<1-\frac{2}{3}=\frac{1}{3} .
$$

By [8], there exist three elements $u_{1}, u_{2}, u_{3} \in U(A)$ such that $\frac{3 a}{M}=u_{1}+u_{2}+u_{3}$. So by the equation (5.4), we have

$$
\begin{aligned}
L(a x) & =\frac{M}{3} L\left(3 \frac{a}{M} x\right)=\frac{M}{3}\left(L\left(u_{1} x\right)+L\left(u_{2} x\right)+L\left(u_{3} x\right)\right) \\
& =\frac{M}{3}\left(u_{1}+u_{2}+u_{3}\right) L(x)=\frac{M}{3} \cdot 3 \frac{a}{M} L(x)=a L(x)
\end{aligned}
$$

for all $a \in A$ and all $x \in X$. Hence

$$
L(a x+b y)=L(a x)+L(b y)=a L(x)+b L(y)
$$

for all $a, b \in A$ and all $x, y \in X$. Thus the unique generalized additive mapping $L: X \rightarrow Y$ is an $A$-linear mapping.

Now we are ready to prove the $C^{*}$-algebra isomorphisms between unital $C^{*}$-algebras.

Assume that $A$ is a unital $C^{*}$-algebra with norm $\|\cdot\|_{A}$ with unit $e$ and $B$ is a unital $C^{*}$-algebra with norm $\|\cdot\|_{B}$. Let $U(A)$ be the set of all unitary elements in $A$. 
Theorem 5.2. Let $h: A \rightarrow B$ be an odd bijective mapping satisfying $h\left(\frac{2^{s}}{r^{s}} u y\right)=$ $h\left(\frac{2^{s}}{r^{s}} u\right) h(y)$ for all $u \in U(A)$, all $y \in A$, and $s=0,1,2, \ldots$, for which there exists a function $\phi: A^{n} \rightarrow[0, \infty)$ such that

$$
\begin{gathered}
\sum_{j=0}^{\infty}\left(\frac{r}{2}\right)^{j} \phi\left(\left(\frac{2}{r}\right)^{j} x_{1}, \ldots,\left(\frac{2}{r}\right)^{j} x_{n}\right)<\infty, \\
\left\|D_{\mu} h\left(x_{1}, \ldots, x_{n}\right)\right\|_{B} \leq \phi\left(x_{1}, \ldots, x_{n}\right), \\
\left\|h\left(\frac{2^{s}}{r^{s}} u^{*}\right)-h\left(\frac{2^{s}}{r^{s}} u\right)^{*}\right\|_{B} \leq \phi\left(\left(\frac{2}{r}\right)^{s} u, \ldots,\left(\frac{2}{r}\right)^{s} u\right)
\end{gathered}
$$

for all $\mu \in S^{1}:=\{\lambda \in \mathbb{C}|| \lambda \mid=1\}$, all $u \in U(A), s=0,1,2, \ldots$, and all $x_{1}, \ldots, x_{n} \in A$. Assume that $\lim _{s \rightarrow \infty} \frac{r^{s}}{2^{s}} h\left(\frac{2^{s}}{r^{s}} e\right)$ is invertible. Then the odd bijective mapping $h: A \rightarrow B$ is a $C^{*}$-algebra isomorphism.

Proof. Consider $C^{*}$-algebra $A$ and $B$ as left Banach modules over the unital $C^{*}$ algebra $\mathbb{C}$. By Theorem 5.1 , there exists a unique generalized $\mathbb{C}$-linear mapping $H: A \rightarrow B$ such that

$$
\|h(x)-H(x)\|_{B} \leq \frac{1}{4_{n-2} C_{\frac{2}{n}-1}} \widetilde{\phi}(x, x, 0, \ldots, 0)
$$

for all $x \in A$, where the generalized additive mapping $H: A \rightarrow B$ is given by

$$
H(x)=\lim _{s \rightarrow \infty} \frac{r^{s}}{2^{s}} h\left(\frac{2^{s}}{r^{s}} x\right)
$$

for all $x \in A$. By equations (5.5) and (5.6), we have

$$
\begin{aligned}
H\left(u^{*}\right) & =\lim _{s \rightarrow \infty} \frac{r^{s}}{2^{s}} h\left(\frac{2^{s}}{r^{s}} u^{*}\right)=\lim _{s \rightarrow \infty} \frac{r^{s}}{2^{s}} h\left(\frac{2^{s}}{r^{s}} u\right)^{*} \\
& =\left(\lim _{s \rightarrow \infty} \frac{r^{s}}{2^{s}} h\left(\frac{2^{s}}{r^{s}} u\right)\right)^{*}=H(u)^{*}
\end{aligned}
$$

for all $u \in U(A)$. Since $H$ is $\mathbb{C}$-linear and each $x \in A$ is a finite linear combination of unitary elements (see [9], Theorem 4.1.7), i.e.,

$$
x=\sum_{j=1}^{m} \lambda_{j} u_{j}
$$

where $\lambda_{i} \in \mathbb{C}, u_{j} \in U(A)$,

$$
\begin{aligned}
H\left(x^{*}\right) & =H\left(\sum_{j=1}^{m} \overline{\lambda_{j}} u_{j}^{*}\right)=\sum_{j=1}^{m} \overline{\lambda_{j}} H\left(u_{j}\right)^{*}=\left(\sum_{j=1}^{m} \lambda_{j} H\left(u_{j}\right)\right)^{*} \\
& =H\left(\sum_{j=1}^{m} \lambda_{j} u_{j}\right)^{*}=H(x)^{*} \text { for every } x \in A .
\end{aligned}
$$


Since $h\left(\frac{2^{s}}{r^{s}} u y\right)=h\left(\frac{2^{s}}{r^{s}} u\right) h(y)$ for all $u \in U(A)$, all $y \in A$, and $s=0,1,2, \ldots$, we have

$$
H(u y)=\lim _{s \rightarrow \infty} \frac{r^{s}}{2^{s}} h\left(\frac{2^{s}}{r^{s}} u y\right)=\lim _{s \rightarrow \infty} \frac{r^{s}}{2^{s}} h\left(\frac{2^{s}}{r^{s}} u\right) h(y)=H(u) h(y)
$$

for all $u \in U(A)$, all $y \in A$. By the additive property of $H$ and above equation,

$$
\frac{2^{s}}{r^{s}} H(u y)=H\left(\frac{2^{s}}{r^{s}} u y\right)=H\left(u\left(\frac{2^{s}}{r^{s}} y\right)\right)=H(u) h\left(\frac{2^{s}}{r^{s}} y\right)
$$

for all $u \in U(A)$, all $y \in A$. Hence we may have that

$$
H(u y)=\frac{r^{s}}{2^{s}} H(u) h\left(\frac{2^{s}}{r^{s}} y\right)=H(u) \frac{r^{s}}{2^{s}} h\left(\frac{2^{s}}{r^{s}} y\right)
$$

for all $u \in U(A)$, all $y \in A$. Now, by taking $s \rightarrow \infty$,

$$
H(u y)=H(u) H(y)
$$

for all $u \in U(A)$, all $y \in A$. Since $H$ is $\mathbb{C}$-linear and each $x \in A$ is a finite linear combination of unitary elements, i.e.,

$$
x=\sum_{j=1}^{m} \lambda_{j} u_{j}
$$

where $\lambda_{i} \in \mathbb{C}, u_{j} \in U(A)$, the equation (5.7) implies that

$$
\begin{aligned}
H(x y) & =H\left(\sum_{j=1}^{m} \lambda_{j} u_{j} y\right)=\sum_{j=1}^{m} \lambda_{j} H\left(u_{j} y\right)=\sum_{j=1}^{m} \lambda_{j} H\left(u_{j}\right) H(y) \\
& =H\left(\sum_{j=1}^{m} \lambda_{j} u_{j}\right) H(y)=H(x) H(y)
\end{aligned}
$$

for all $x, y \in A$. Also, we have $H(e) H(y)=H(e y)=H(e) h(y)$ for all $y \in A$. By the assumption that $\lim _{s \rightarrow \infty} \frac{r^{s}}{2^{s}} h\left(\frac{2^{s}}{r^{s}} e\right)=H(e)$ is invertible,

$$
H(y)=h(y)
$$

for all $y \in A$. Thus the odd bijective mapping $h: A \rightarrow B$ is a $C^{*}$-algebra isomorphism.

Corollary 5.3. Let $h: A \rightarrow B$ be an odd bijective mapping satisfying $h\left(\frac{2^{s}}{r^{s}} u y\right)=$ $h\left(\frac{2^{s}}{r^{s}} u\right) h(y)$ for all $u \in U(A)$, all $y \in A$, and $s=0,1,2, \ldots$, for which there exists a function $\phi: A^{n} \rightarrow[0, \infty)$ such that

$$
\begin{aligned}
& \sum_{j=0}^{\infty}\left(\frac{r}{2}\right)^{j} \phi\left(\left(\frac{2}{r}\right)^{j} x_{1}, \ldots,\left(\frac{2}{r}\right)^{j} x_{n}\right)<\infty, \\
& \left\|D_{\mu} h\left(x_{1}, \ldots, x_{n}\right)\right\|_{B} \leq \phi\left(x_{1}, \ldots, x_{n}\right),
\end{aligned}
$$




$$
\left\|h\left(\frac{2^{s}}{r^{s}} u^{*}\right)-h\left(\frac{2^{s}}{r^{s}} u\right)^{*}\right\|_{B} \leq \phi\left(\left(\frac{2}{r}\right)^{j} u, \ldots,\left(\frac{2}{r}\right)^{j} u\right)
$$

for all $\mu=1, i$, all $u \in U(A), s=0,1,2, \ldots$, and all $x_{1}, \ldots, x_{n} \in A$. Assume that $\lim _{s \rightarrow \infty} \frac{r^{s}}{2^{s}} h\left(\frac{2^{s}}{r^{s}} e\right)$ is invertible. If $h(t x)$ is continuous in $\mathbb{R}$ for each fixed $x \in A$, then the odd bijective mapping $h: A \rightarrow B$ is a $C^{*}$-algebra isomorphism.

Proof. Let $\mu=1$ in the equation (5.9). By Theorem 5.1, there exists a unique generalized $\mathbb{C}$-linear mapping $H: A \rightarrow B$ satisfying the equation (5.3). By the same reasoning as in the proof of [11], the generalized additive mapping $H: A \rightarrow B$ is $\mathbb{R}$-linear. Now, let $\mu=i$ in the equation (5.9). Similar to the proof of Theorem 5.1, we have

$$
H(i x)=\lim _{s \rightarrow \infty} \frac{r^{s}}{2^{s}} h\left(\frac{2^{s}}{r^{s}} i x\right)=\lim _{s \rightarrow \infty} \frac{r^{s} i}{2^{s}} h\left(\frac{2^{s}}{r^{s}} x\right)=i H(x)
$$

for all $x \in A$. For each element $\lambda \in \mathbb{C}, \lambda=s+i t$, where $s, t \in \mathbb{R}$. Hence

$$
H(\lambda x)=H(s x+i t x)=(s+i t) H(x)=\lambda H(x)
$$

for all $\lambda \in \mathbb{C}$, and all $x \in A$. Thus

$$
H(\zeta x+\eta y)=\zeta H(x)+\eta H(y)
$$

for all $\zeta, \eta \in \mathbb{C}$, and all $x, y \in A$. Hence the generalized additive mapping $H: A \rightarrow B$ is $\mathbb{C}$-linear. The remains of the proof is similar to the proof of Theorem 5.2.

\section{References}

[1] J.-H. Bae and W.-G. Park, On the generalized Hyers-Ulam-Rassias stability in Banach modules over a $C^{*}$-algebra, J. Math. Anal. Appl. 294 (2004), no. 1, 196-205.

[2] C. Baak, D.-H. Boo, Th. M. Rassias, Generalized additive mapping in Banach modules and isomorphisms between $C^{*}$-algebras, J. Math. Anal. Appl. 314 (2006), no. 1, 150161.

[3] H. Y. Chu and D. S. Kang, On the stability of an $n$-dimensional cubic functional equation, J. Math. Anal. Appl. 325 (2007), no. 1, 595-607.

[4] G.-L. Forti, Hyers-Ulam stability of functional equations in several variables, Aequationes Math. 50 (1995), no. 1-2, 143-190.

[5] _ Comments on the core of the direct method for proving Hyers-Ulam stability of functional equations, J. Math. Anal. Appl. 295 (2004), no. 1, 127-133.

[6] P. Găvruta, A generalization of the Hyers-Ulam-Rassias stability of approximately additive mappings, J. Math. Anal. Appl. 184 (1994), no. 3, 431-436.

[7] D. H. Hyers, On the stability of the linear functional equation, Proc. Nat. Acad. Sci. U.S.A. 27 (1941), 222-224.

[8] R. V. Kadison and G. Pedersen, Means and convex combinations of unitary operators, Math. Scand. 57 (1985), no. 2, 249-266.

[9] R. V. Kadison and J. R. Ringrose, Fundamentals of the Theory of Operator Algebras. Vol. I, Elementary theory. Pure and Applied Mathematics, 100. Academic Press, Inc. [Harcourt Brace Jovanovich, Publishers], New York, 1983.

[10] S.-H. Lee, H. Koh, and S.-H. Ku, Investigation of the stability via shadowing property, J. Inequal. Appl. 2009 (2009), Art. ID 156167, 12 pp. 
[11] Th. M. Rassias, On the stability of the linear mapping in Banach spaces, Proc. Amer. Math. Soc. 72 (1978), no. 2, 297-300.

[12] F. Skof, Local properties and approximation of operators, Rend. Sem. Mat. Fis. Milano 53 (1983), 113-129.

[13] J. Tabor, Locally expanding mappings and hyperbolicity, Topol. Methods Nonlinear Anal. 30 (2007), no. 2, 335-343

[14] J. Tabor and J. Tabor, General stability of functional equations of linear type, J. Math. Anal. Appl. 328 (2007), no. 1, 192-200.

[15] S. M. Ulam, Problems in Morden Mathematics, Wiley, New York, 1960.

\section{HAHNG-YUN CHU}

Department of Mathematics

Chungnam National University

DAEJEON 305-764, Korea

E-mail address: hychu@cnu.ac.kr

GilJun Han

Department of Mathematics Education

DANKOOK UNIVERSITY

YoNGIN 448-701, KoreA

E-mail address: gilhan@dankook.ac.kr

Dong Seung Kang

Department of Mathematics Education

DANKOOK UNIVERSITY

YONGIN 448-701, KOREA

E-mail address: dskang@dankook.ac.kr 